\title{
On a Kirchhoff diffusion equation with integral condition
}

\author{
Danh Hua Quoc Nam ${ }^{1}$, Dumitru Baleanu ${ }^{2,3,4}$, Nguyen Hoang Luc ${ }^{5}$ and Nguyen Huu Can ${ }^{6 *}$
}

\section{"Correspondence:}

nguyenhuucan@tdtu.edu.vn

${ }^{6}$ Applied Analysis Research Group,

Faculty of Mathematics and

Statistics, Ton Duc Thang University,

Ho Chi Minh City, Vietnam

Full list of author information is

available at the end of the article

\begin{abstract}
This paper is devoted to Kirchhoff-type parabolic problem with nonlocal integral condition. Our problem has many applications in modeling physical and biological phenomena. The first part of our paper concerns the local existence of the mild solution in Hilbert scales. Our results can be studied into two cases: homogeneous case and inhomogeneous case. In order to overcome difficulties, we applied Banach fixed point theorem and some new techniques on Sobolev spaces. The second part of the paper is to derive the ill-posedness of the mild solution in the sense of Hadamard.
\end{abstract}

MSC: $35 \mathrm{R} 11 ; 35 \mathrm{~B} 65 ; 26 \mathrm{~A} 33$

Keywords: Kirchhoff-type problems; Nonlocal problem; Well-posedness; Regularization

\section{Introduction}

Kirchhoff problems have been considerably investigated recently, see [1-6]. The topic of Kirchhoff-type models arises from their contributions to the modeling of many physical and biological phenomena. Kirchhoff-type problems also appear in reaction-diffusion equations that concern population density. For more applications of such modeling and Kirchhoff-type problems, we refer to [7]. In our paper, we consider the following Kirchhoff-type problem for parabolic equation:

$$
\begin{cases}\partial_{t} u(x, t)=\mathcal{M}\left(\|u\|_{L^{2}}\right) \Delta u+F(x, t), & (x, t) \in \Omega \times(0, T), \\ u(x, t)=0, & x \in \partial \Omega, t \in(0, T]\end{cases}
$$

with the following nonlocal condition:

$$
\alpha u(x, 0)+\beta \int_{0}^{T} u(x, t) d t=\psi(x), \quad x \in \Omega
$$

Here $\mathcal{M} \in C^{1}(\mathbb{R})$ is a function satisfying $m_{0} \leq \mathcal{M}(s) \leq \bar{m}_{0} \forall s \in \mathbb{R}$. Moreover, we assume that there exists $K>0$ such that $|\mathcal{M}(s)-\mathcal{M}(t)| \leq K|s-t|, s, t \in \mathbb{R}$. The nonlocal problem

(c) The Author(s) 2020. This article is licensed under a Creative Commons Attribution 4.0 International License, which permits use, sharing, adaptation, distribution and reproduction in any medium or format, as long as you give appropriate credit to the original author(s) and the source, provide a link to the Creative Commons licence, and indicate if changes were made. The images or other third party material in this article are included in the article's Creative Commons licence, unless indicated otherwise in a credit line to the material. If material is not included in the article's Creative Commons licence and your intended use is not permitted by statutory regulation or exceeds the permitted use, you will need to obtain permission directly from the copyright holder. To view a copy of this licence, visit http://creativecommons.org/licenses/by/4.0/. 
as above was first considered in [8], where Chipot et al. focused on the following equation:

$$
\partial_{t} u(x, t)=\mathcal{M}\left(\int_{\Omega} u d x\right) \Delta u+F(x, t) .
$$

One application of equation (1.3) is to model the density of a population of bacteria; it also appears when investigating heat propagation or in epidemic theory.

In [8], the authors also studied a nonlocal problem as follows:

$$
\begin{cases}u_{t}(x, t)-\mathcal{M}(\ell(u)(t)) \Delta u=F(x, t), & \text { in } \Omega \times(0, T), \\ u=0, & \text { on } \Sigma, \\ u(x, 0)=u_{0}(x), & \text { in } \Omega \times\{T\} .\end{cases}
$$

Here $\ell: L^{2}(\Omega) \rightarrow \mathbb{R}$ is a continuous linear form. The function $u$ is here the density of a population located at $x$ at the time $t, F$ is the external source, $\mathcal{M}$ is the diffusion rate. Very recently, Ferreira et al. [9] have studied a model with nonlocal coupled diffusivity terms

$$
\begin{cases}u_{t}-\mathcal{D}_{1}(p(u)(t), q(v)(t)) \Delta u=f_{1}(u, v), & \text { in } Q_{T}, \\ v_{t}-\mathcal{D}_{2}(r(u)(t), s(v)(t)) \Delta v=f_{2}(u, v), & \text { in } Q_{T}, \\ u=u_{0}, \quad v=v_{0}, & \text { in } \Omega \times\{0\} .\end{cases}
$$

Although initial problems have been investigated by many authors, there are very few papers for the inverse problems of a nonlocal parabolic equation. In [10], the authors consider the backward in time nonlocal nonlinear parabolic equation as follows

$$
\begin{cases}u_{t}(x, t)-\mathcal{D}(\ell(u)(t)) \Delta u=F(u, x, t), & \text { in } \Omega \times(0, T), \\ \frac{\partial u}{\partial \sigma}=0, & \text { on } \Sigma, \\ u(x, T)=g(x), & \text { in } \Omega \times\{T\} .\end{cases}
$$

Very recently, Tuan, Nam, and Nhat [11] first studied a terminal value problem for Kirchhoff's model of parabolic type as follows:

$$
\begin{cases}\partial_{t} u(x, t)=\mathcal{M}\left(\|u\|_{L^{2}}\right) \Delta u+F(x, t), & (x, t) \in \Omega \times(0, T), \\ u(x, t)=0, & x \in \partial \Omega, t \in(0, T], \\ u(x, T)=g(x) & x \in \partial \Omega .\end{cases}
$$

If $\alpha=0, \beta=1$, problem (1.1) and some similar models have been recently considered by N. Dokuchaev [12, 13], S.I. Volodymyr et al. [14], S.L. Pulkina et al. [15], and in the references therein. Besides, there are many works which focused on this topic; see, e.g., [16-27].

For the two problems above, the authors showed that they are ill-posed and then focused on their regularization methods. Our paper is motivated by the recent paper [12] in which the authors considered the nonlocal in time condition replacing the usual Cauchy conditions, that is,

$$
\alpha u(x, 0)+\beta \int_{0}^{T} u(x, t) d t=\psi(x) .
$$


The problem for this equation with the Cauchy condition $u(x, 0) \equiv g(x)$ at the initial time $t=0$ is well-posed in the usual classes of solutions. In contrast, the problem with the Cauchy condition $u(x, T) \equiv g(x)$ at the terminal time $t=T$ is ill-posed. This means that a prescribed profile of temperature at time $t=T$ cannot be achieved via an appropriate selection of the initial temperature. Respectively, the initial temperature profile cannot be recovered from the observed temperature at the terminal time. In particular, the process is not robust with respect to small deviations of its terminal profile $u(\cdot, T)$. This makes this problem ill-posed, despite the fact that solvability and uniqueness can still be achieved for some very smooth analytical boundary data or for a special selection of the domains. We can interpret this as the existence of a diffusion with a prescribed average over a time interval. In addition, this can be interpreted as solvability of the following inverse problem: given $\int_{0}^{T} u(x, t) d t$ for all $x \in \Omega$, recover the entire process $u(x, t)$ on $\Omega \times(0, T)$.

Our main results in this paper are described as follows:

- The first part focuses on the local existence of a mild solution.

- The second part gives the ill-posedness of our problem in the simple case $F=0, \alpha=0$. To the best of our knowledge, our results concerning the nonlocal condition for Kirchhoff diffusion equation have not been investigated, yet.

This paper is organized as follows. Section 2 introduces some preliminaries and mild solutions of our problem. Section 3 derives the well-posedness of the mild solution in the homogeneous case. In Sect. 4, we extend the results of Sect. 3 to the inhomogeneous case. Finally, in Sect. 5, we show the ill-posedness of the mild solution.

\section{Preliminaries}

Let us introduce a few properties of the eigenvalues of the operator $-\Delta$, see [6]. We have the following equality:

$$
-\Delta \varphi_{n}(x)=-\lambda_{n} \varphi_{n}(x), \quad x \in \Omega ; \quad \varphi_{n}=0, \quad x \in \partial \Omega, n \in \mathbb{N},
$$

where $\left\{\lambda_{n}\right\}_{n=1}^{\infty}$ is called the set of eigenvalues of $-\Delta$ satisfying

$$
0<\lambda_{1} \leq \lambda_{2} \leq \cdots \leq \lambda_{n} \leq \cdots,
$$

and $\lim _{n \rightarrow \infty} \lambda_{n}=\infty$. For any $q \geq 0$, we also define the space

$$
H^{q}(\Omega)=\left\{u \in L_{2}(\Omega): \sum_{n=1}^{\infty} \lambda_{n}^{2 q}\left|\left(u, \varphi_{n}\right)\right|^{2}<\infty\right\},
$$

then $H^{q}(\Omega)$ is a Hilbert space endowed with the norm

$$
\|u\|_{H^{q}(\Omega)}=\left(\sum_{n=1}^{\infty} \lambda_{n}^{2 q}\left|\left(u, e_{n}\right)\right|^{2}\right)^{\frac{1}{2}} .
$$

Lemma 2.1 The following inclusions hold true:

$$
\left.\begin{array}{l}
L^{p}(\Omega) \hookrightarrow D\left(\mathcal{A}^{\sigma}\right), \quad \text { if }-\frac{d}{4}<\sigma \leq 0, p \geq \frac{2 d}{d-4 \sigma}, \\
D\left(\mathcal{A}^{\sigma}\right) \hookrightarrow L^{p}(\Omega), \quad \text { if } 0 \leq \sigma<\frac{d}{4}, p \leq \frac{2 d}{d-4 \sigma} .
\end{array}\right\}
$$


For $r \geq 0$ and $v \in L^{\infty}\left((0, T) ; D\left(\mathcal{A}^{r}\right)\right)$, we denote

$$
\|v\|_{r}=\underset{0 \leq t \leq T}{\operatorname{esssup}}\|v(t)\|_{D\left(\mathcal{A}^{r}\right)}
$$

\subsection{The mild solution of our problem}

Let us assume that problem (1.1) has a unique solution $u$. Assume that the exact $u$ is given by a Fourier series

$$
u(t, x)=\sum_{n=1}^{\infty} u_{n}(t) \varphi_{n}(x), \quad \text { with } u_{n}(t)=\left\langle u(t, \cdot), \varphi_{n}(\cdot)\right\rangle
$$

Multiplying both sides of (1.1) by the term $\exp \left(\int_{0}^{t} \lambda_{n} \mathcal{M}\left(\|u(\cdot, s)\|_{L^{2}}\right) d s\right)$, we get that

$$
\begin{aligned}
u_{n}(t)= & \exp \left(-\lambda_{n} \int_{0}^{t} \mathcal{M}\left(\|u(\cdot, s)\|_{L^{2}}\right) d s\right) u_{n}(0) \\
& +\int_{0}^{t} \exp \left(-\lambda_{n} \int_{s}^{t} \mathcal{M}\left(\|u(\cdot, \eta)\|_{L^{2}}\right) d \eta\right) F_{n}(s) d s .
\end{aligned}
$$

From the condition $\alpha u(x, 0)+\beta \int_{0}^{T} u(x, t) d t=\psi(x)$, we know that

$$
\alpha u_{n}(0)+\beta \int_{0}^{T} u_{n}(t) d t=\psi_{n}
$$

Therefore, we obtain that

$$
\begin{aligned}
& \left(\alpha+\beta \int_{0}^{T} \exp \left(-\lambda_{n} \int_{0}^{t} \mathcal{M}\left(\|u(\cdot, s)\|_{L^{2}}\right) d s\right) d t\right) u_{n}(0) \\
& \quad+\beta \int_{0}^{T} \int_{0}^{t} \exp \left(-\lambda_{n} \int_{s}^{t} \mathcal{M}\left(\|u(\cdot, \eta)\|_{L^{2}}\right) d \eta\right) F_{n}(s) d s d t=\psi_{n} .
\end{aligned}
$$

This gives that

$$
u_{n}(0)=\frac{\psi_{n}-\beta \int_{0}^{T} \int_{0}^{t} \exp \left(-\lambda_{n} \int_{s}^{t} \mathcal{M}\left(\|u(\cdot, \eta)\|_{L^{2}}\right) d \eta\right) F_{n}(s) d s d t}{\alpha+\beta \int_{0}^{T} \exp \left(-\lambda_{n} \int_{0}^{t} \mathcal{M}\left(\|u(\cdot, s)\|_{L^{2}}\right) d s\right) d t} .
$$

The latter equality, together with expression (2.7), yields that

$$
\begin{aligned}
u_{n}(t)= & \frac{\exp \left(-\lambda_{n} \int_{0}^{t} \mathcal{M}\left(\|u(\cdot, s)\|_{L^{2}}\right) d s\right) \psi_{n}}{\alpha+\beta \int_{0}^{T} \exp \left(-\lambda_{n} \int_{0}^{t} \mathcal{M}\left(\|u(\cdot, s)\|_{L^{2}}\right) d s\right) d t} \\
& -\beta \frac{\exp \left(-\lambda_{n} \int_{0}^{t} \mathcal{M}\left(\|u(\cdot, s)\|_{L^{2}}\right) d s\right) \int_{0}^{T} \int_{0}^{t} \exp \left(-\lambda_{n} \int_{s}^{t} \mathcal{M}\left(\|u(\cdot, \eta)\|_{L^{2}}\right) d \eta\right) F_{n}(s) d s d t}{\alpha+\beta \int_{0}^{T} \exp \left(-\lambda_{n} \int_{0}^{t} \mathcal{M}\left(\|u(\cdot, s)\|_{L^{2}}\right) d s\right) d t} \\
& +\int_{0}^{t} \exp \left(-\lambda_{n} \int_{s}^{t} \mathcal{M}\left(\|u(\cdot, \eta)\|_{L^{2}}\right) d \eta\right) F_{n}(s) d s
\end{aligned}
$$

Let us define the following operators:

$$
\Phi_{1} u(t)=\sum_{n=1}^{\infty} \frac{\exp \left(-\lambda_{n} \int_{0}^{t} \mathcal{M}\left(\|u(\cdot, s)\|_{L^{2}}\right) d s\right) \psi_{n}}{\alpha+\beta \int_{0}^{T} \exp \left(-\lambda_{n} \int_{0}^{t} \mathcal{M}\left(\|u(\cdot, s)\|_{L^{2}}\right) d s\right) d t} \varphi_{n}
$$




$$
\begin{aligned}
& \Phi_{2} u(t) \\
& =\beta \sum_{n=1}^{\infty} \frac{\exp \left(-\lambda_{n} \int_{0}^{t} \mathcal{M}\left(\|u(\cdot, s)\|_{L^{2}}\right) d s\right) \int_{0}^{T} \int_{0}^{t} \exp \left(-\lambda_{n} \int_{s}^{t} \mathcal{M}\left(\|u(\cdot, \eta)\|_{L^{2}}\right) d \eta\right) F_{n}(s) d s d t}{\alpha+\beta \int_{0}^{T} \exp \left(-\lambda_{n} \int_{0}^{t} \mathcal{M}\left(\|u(\cdot, s)\|_{L^{2}}\right) d s\right) d t} \varphi_{n},
\end{aligned}
$$

and

$$
\Phi_{3} u(t)=\sum_{n=1}^{\infty}\left(\int_{0}^{t} \exp \left(-\lambda_{n} \int_{s}^{t} \mathcal{M}\left(\|u(\cdot, \eta)\|_{L^{2}}\right) d \eta\right) F_{n}(s) d s\right) \varphi_{n}
$$

The above three definitions lead to

$$
u(t)=\Phi_{1} u(t)+\Phi_{2} u(t)+\Phi_{3} u(t)
$$

\section{The existence of a mild solution in the homogeneous case}

In this section, we derive the existence and uniqueness of the mild solution in the case of $F=0$. We will show that the following integral equation:

$$
u(t)=\sum_{n=1}^{\infty} \frac{\exp \left(-\lambda_{n} \int_{0}^{t} \mathcal{M}\left(\|u(\cdot, s)\|_{L^{2}}\right) d s\right) \psi_{n}}{\alpha+\beta \int_{0}^{T} \exp \left(-\lambda_{n} \int_{0}^{t} \mathcal{M}\left(\|u(\cdot, s)\|_{L^{2}}\right) d s\right) d t} \varphi_{n}
$$

has a unique solution.

\section{Theorem 3.1}

(a) Let $\alpha>0, \beta>0$, and $\psi \in D\left(\mathcal{A}^{r+1}\right)$. If $T$ is small enough, problem (3.1) has a unique solution $u \in L^{\infty}\left(0, T ; D\left(\mathcal{A}^{r}\right)\right)$.

(b) Let $\alpha=0, \beta>0$, and $\psi \in D\left(\mathcal{A}^{r+2}\right)$. If $T$ is small enough, problem (3.1) has a unique solution $u \in L^{\infty}\left(0, T ; D\left(\mathcal{A}^{r}\right)\right)$.

Remark 3.1 The property of global existence for the mild solution of problem (3.1) is an open problem and is more difficult. We will discuss it in future works.

Proof We will show that

$$
\Phi_{1} u=u
$$

by using Banach fixed point theorem. Now, we divide the proof into two parts.

Part 1. Estimate the term $\left\|\Phi_{1} u-\Phi_{1} v\right\|_{L_{a}^{\infty}\left(0, T ; D\left(\mathcal{A}^{r}\right)\right)}$ in the case $\alpha>0, \beta>0$

First, we get that

$$
\begin{gathered}
\left\|\Phi_{1} u-\Phi_{1} v\right\|_{D\left(\mathcal{A}^{r}\right)}^{2} \\
=\sum_{n=1}^{\infty} \lambda_{n}^{2 r}\left(\frac{\exp \left(-\lambda_{n} \int_{0}^{t} \mathcal{M}\left(\|u(\cdot, s)\|_{L^{2}}\right) d s\right)}{\alpha+\beta \int_{0}^{T} \exp \left(-\lambda_{n} \int_{0}^{t} \mathcal{M}\left(\|u(\cdot, s)\|_{L^{2}}\right) d s\right) d t}\right. \\
\left.\quad-\frac{\exp \left(-\lambda_{n} \int_{0}^{t} \mathcal{M}\left(\|v(\cdot, s)\|_{L^{2}}\right) d s\right)}{\alpha+\beta \int_{0}^{T} \exp \left(-\lambda_{n} \int_{0}^{t} \mathcal{M}\left(\|v(\cdot, s)\|_{L^{2}}\right) d s\right) d t}\right)^{2} \psi_{n}^{2} \\
\leq 2 \alpha^{2}
\end{gathered}
$$




$$
\begin{aligned}
& \times \sum_{n=1}^{\infty} \lambda_{n}^{2 r} \frac{\left(\mathcal{H}_{1}(u, v)\right)^{2}}{\left(\alpha+\beta \int_{0}^{T} \exp \left(-\lambda_{n} \int_{0}^{t} \mathcal{M}\left(\|u(\cdot, s)\|_{L^{2}}\right) d s\right) d t\right)^{2}\left(\alpha+\beta \int_{0}^{T} \exp \left(-\lambda_{n} \int_{0}^{t} \mathcal{M}\left(\|v(\cdot, s)\|_{L^{2}}\right) d s\right) d t\right)^{2}} \psi_{n}^{2} \\
& +2 \beta^{2} \\
& \times \sum_{n=1}^{\infty} \lambda_{n}^{2 r} \frac{\left(\mathcal{H}_{2}(u, v)\right)^{2}}{\left(\alpha+\beta \int_{0}^{T} \exp \left(-\lambda_{n} \int_{0}^{t} \mathcal{M}\left(\|u(\cdot, s)\|_{L^{2}}\right) d s\right) d t\right)^{2}\left(\alpha+\beta \int_{0}^{T} \exp \left(-\lambda_{n} \int_{0}^{t} \mathcal{M}\left(\|v(\cdot, s)\|_{L^{2}}\right) d s\right) d t\right)^{2}} \psi_{n}^{2} \\
= & J_{1}+J_{2},
\end{aligned}
$$

where we denote

$$
\begin{aligned}
& \mathcal{H}_{1, n}(u, v)=\exp \left(-\lambda_{n} \int_{0}^{t} \mathcal{M}\left(\|u(\cdot, s)\|_{L^{2}}\right) d s\right)-\exp \left(-\lambda_{n} \int_{0}^{t} \mathcal{M}\left(\|v(\cdot, s)\|_{L^{2}}\right) d s\right) \\
& \mathcal{H}_{2, n}(u, v) \\
& =\exp \left(-\lambda_{n} \int_{0}^{t} \mathcal{M}\left(\|u(\cdot, s)\|_{L^{2}}\right) d s\right) \int_{0}^{T} \exp \left(-\lambda_{n} \int_{0}^{t} \mathcal{M}\left(\|v(\cdot, s)\|_{L^{2}}\right) d s\right) d t \\
& \quad-\exp \left(-\lambda_{n} \int_{0}^{t} \mathcal{M}\left(\|v(\cdot, s)\|_{L^{2}}\right) d s\right) \int_{0}^{T} \\
& \quad \times \exp \left(-\lambda_{n} \int_{0}^{t} \mathcal{M}\left(\|u(\cdot, s)\|_{L^{2}}\right) d s\right) d t
\end{aligned}
$$

First, applying the inequality $\left|e^{-y}-e^{-z}\right| \leq|y-z|$, we estimate $\mathcal{H}_{1}$ as follows:

$$
\begin{aligned}
\left|\mathcal{H}_{1, n}(u, v)\right| & \leq \lambda_{n} \int_{0}^{t}\left(\mathcal{M}\left(\|u(\cdot, s)\|_{L^{2}}\right)-\mathcal{M}\left(\|v(\cdot, s)\|_{L^{2}}\right)\right) d s \\
& \leq K \lambda_{n} \int_{0}^{t}\|u-v\|_{L^{2}}^{2} d s .
\end{aligned}
$$

The latter inequality leads to

$$
\begin{aligned}
J_{1} & \leq \frac{2}{\alpha^{2}} \sum_{n=1}^{\infty}\left|\mathcal{H}_{1, n}(u, v)\right|^{2} \lambda_{n}^{2 r} \psi_{n}^{2} \\
& \leq \frac{2 K^{2}}{\alpha^{2}} \sum_{n=1}^{\infty} \lambda_{n}^{2 r+2} \psi_{n}^{2}\left(\int_{0}^{t}\|u-v\|_{L^{2}}^{2} d s\right) \\
& \leq \frac{2 K^{2} T}{\alpha^{2}}\|\psi\|_{D\left(\mathcal{A}^{r+1}\right)}^{2}\|u-v\|_{r}^{2} .
\end{aligned}
$$

Noting that $m_{0} \leq \mathcal{M}(z) \leq \bar{m}_{0}$, we get that

$$
\begin{aligned}
\mathcal{H}_{2, n}(u, v) & \\
\leq & \exp \left(-\lambda_{n} \int_{0}^{t} \mathcal{M}\left(\|u(\cdot, s)\|_{L^{2}}\right) d s\right) \\
\quad & \left(\int_{0}^{T} \exp \left(-\lambda_{n} \int_{0}^{t} \mathcal{M}\left(\|v(\cdot, s)\|_{L^{2}}\right) d s\right) d t\right. \\
& \left.-\int_{0}^{T} \exp \left(-\lambda_{n} \int_{0}^{t} \mathcal{M}\left(\|u(\cdot, s)\|_{L^{2}}\right) d s\right) d t\right)
\end{aligned}
$$




$$
\begin{aligned}
& +\int_{0}^{T} \exp \left(-\lambda_{n} \int_{0}^{t} \mathcal{M}\left(\|u(\cdot, s)\|_{L^{2}}\right) d s\right) d t \\
& \quad \times\left(\exp \left(-\lambda_{n} \int_{0}^{t} \mathcal{M}\left(\|u(\cdot, s)\|_{L^{2}}\right) d s\right)-\exp \left(-\lambda_{n} \int_{0}^{t} \mathcal{M}\left(\|v(\cdot, s)\|_{L^{2}}\right) d s\right)\right) \\
& \leq \int_{0}^{T} \lambda_{n} \int_{0}^{t}\left(\mathcal{M}\left(\|v(\cdot, s)\|_{L^{2}}\right)-\mathcal{M}\left(\|u(\cdot, s)\|_{L^{2}}\right)\right) d s d t \\
& +T \lambda_{n} \int_{0}^{t}\left(\mathcal{M}\left(\|v(\cdot, s)\|_{L^{2}}\right)-\mathcal{M}\left(\|u(\cdot, s)\|_{L^{2}}\right)\right) d s .
\end{aligned}
$$

Since $\mathcal{M}$ is globally Lipschitz and noting the Sobolev embedding $D\left(\mathcal{A}^{r}\right) \hookrightarrow L^{2}(\Omega)$, we derive that

$$
\begin{aligned}
& \left|\mathcal{H}_{2, n}(u, v)\right|^{2} \\
& \quad \leq K^{2} \lambda_{n}^{2}\left(\int_{0}^{T} \int_{0}^{t}\|u(\cdot, s)-v(\cdot, s)\|_{L^{2}} d s d t\right)^{2}+T^{2} \lambda_{n}^{2}\left(\int_{0}^{t}\|u(\cdot, s)-v(\cdot, s)\|_{L^{2}} d s\right)^{2} \\
& \quad \leq\left(K^{2} \lambda_{n}^{2} T^{3}+T^{3} \lambda_{n}^{2}\right)\|u-v\|_{r}^{2} .
\end{aligned}
$$

Here we have applied Hölder inequality. Combining (3.2), (3.6), and (3.8), we get that

$$
\left\|\Phi_{1} u-\Phi_{1} v\right\|_{D\left(\mathcal{A}^{r}\right)}^{2} \leq \bar{C}_{1}(T)\|\psi\|_{D\left(\mathcal{A}^{r+1}\right)}^{2}\|u-v\|_{a, r}^{2},
$$

where we denote $\bar{C}_{1}(T)=2 \beta^{2}\left(K^{2} T^{3}+T^{3}\right)+\frac{2 K^{2} T}{\alpha^{2}}$. Since the left-hand side of (3.9) is independent of $t$, we know that

$$
\left\|\Phi_{1} u-\Phi_{1} v\right\|_{r}^{2} \leq \bar{C}_{1}(T)\|\psi\|_{D\left(\mathcal{A}^{r+1}\right)}^{2}\|u-v\|_{r}^{2}
$$

By choosing $T$ small enough, we can deduce that $\Phi_{1}$ is a contraction on $L^{\infty}\left(0, T ; D\left(\mathcal{A}^{r}\right)\right)$. We only show that if $v_{0}=0$ then $\Phi_{1} v_{0} \in L^{\infty}\left(0, T ; D\left(\mathcal{A}^{r}\right)\right)$. Indeed,

$$
\begin{aligned}
\Phi_{1} v_{0} & =\sum_{n=1}^{\infty} \frac{\exp \left(-\lambda_{n} \int_{0}^{t} \mathcal{M}\left(\|v(\cdot, s)\|_{L^{2}}\right) d s\right) \psi_{n}}{\alpha+\beta \int_{0}^{T} \exp \left(-\lambda_{n} \int_{0}^{t} \mathcal{M}\left(\|v(\cdot, s)\|_{L^{2}}\right) d s\right) d t} \varphi_{n} \\
& =\frac{1}{\alpha+\beta T} \sum_{n=1}^{\infty} \psi_{n} \varphi_{n}=\frac{\psi}{\alpha+\beta T} .
\end{aligned}
$$

Since $\psi \in D\left(\mathcal{A}^{r+1}\right)$, we know that $\Phi_{1} v_{0} \in L^{\infty}\left(0, T ; D\left(\mathcal{A}^{r}\right)\right)$. Based on the previous observations, we deduce that $\Phi_{1} v=v$ has a fixed point $u$. So, we conclude that problem (3.1) has a unique solution $u \in L^{\infty}\left(0, T ; D\left(\mathcal{A}^{r}\right)\right)$.

Part 2. Estimate the term $\left\|\Phi_{1} u-\Phi_{1} v\right\|_{L_{a}^{\infty}\left(0, T ; D\left(\mathcal{A}^{r}\right)\right)}$ in the case $\alpha=0, \beta>0$.

First, we get that

$$
\begin{gathered}
\left\|\Phi_{1} u-\Phi_{1} v\right\|_{D\left(\mathcal{A}^{r}\right)}^{2} \\
=\sum_{n=1}^{\infty} \lambda_{n}^{2 r}\left(\frac{\exp \left(-\lambda_{n} \int_{0}^{t} \mathcal{M}\left(\|u(\cdot, s)\|_{L^{2}}\right) d s\right)}{\beta \int_{0}^{T} \exp \left(-\lambda_{n} \int_{0}^{t} \mathcal{M}\left(\|u(\cdot, s)\|_{L^{2}}\right) d s\right) d t}\right. \\
\left.\quad-\frac{\exp \left(-\lambda_{n} \int_{0}^{t} \mathcal{M}\left(\|v(\cdot, s)\|_{L^{2}}\right) d s\right)}{\beta \int_{0}^{T} \exp \left(-\lambda_{n} \int_{0}^{t} \mathcal{M}\left(\|v(\cdot, s)\|_{L^{2}}\right) d s\right) d t}\right)^{2} \psi_{n}^{2}
\end{gathered}
$$




$$
\begin{aligned}
\leq & \frac{2 \alpha^{2}}{\beta^{4}} \\
& \times \sum_{n=1}^{\infty} \lambda_{n}^{2 r} \frac{\left(\mathcal{H}_{1}(u, v)\right)^{2}}{\left(\int_{0}^{T} \exp \left(-\lambda_{n} \int_{0}^{t} \mathcal{M}\left(\|u(\cdot, s)\|_{L^{2}}\right) d s\right) d t\right)^{2}\left(\int_{0}^{T} \exp \left(-\lambda_{n} \int_{0}^{t} \mathcal{M}\left(\|v(\cdot, s)\|_{L^{2}}\right) d s\right) d t\right)^{2}} \psi_{n}^{2} \\
& +\frac{2}{\beta^{2}} \\
& \times \sum_{n=1}^{\infty} \lambda_{n}^{2 r} \frac{\left(\mathcal{H}_{2}(u, v)\right)^{2}}{\left(\int_{0}^{T} \exp \left(-\lambda_{n} \int_{0}^{t} \mathcal{M}\left(\|u(\cdot, s)\|_{L^{2}}\right) d s\right) d t\right)^{2}\left(\int_{0}^{T} \exp \left(-\lambda_{n} \int_{0}^{t} \mathcal{M}\left(\|v(\cdot, s)\|_{L^{2}}\right) d s\right) d t\right)^{2}} \psi_{n}^{2} \\
= & \bar{J}_{1}+\bar{J}_{2} .
\end{aligned}
$$

Since $\mathcal{M}(z) \leq \bar{m}_{0}$, we get two following estimates:

$$
\begin{aligned}
\int_{0}^{T} \exp \left(-\lambda_{n} \int_{0}^{t} \mathcal{M}\left(\|u(\cdot, s)\|_{L^{2}}\right) d s\right) d t & \geq \bar{m}_{0} \int_{0}^{T} \exp \left(-\lambda_{n} \int_{0}^{t} d s\right) d t \\
& =\bar{m}_{0} \int_{0}^{T} e^{-\lambda_{n} t} d t=\frac{\bar{m}_{0}\left(1-e^{-T \lambda_{n}}\right)}{\lambda_{n}}
\end{aligned}
$$

and

$$
\int_{0}^{T} \exp \left(-\lambda_{n} \int_{0}^{t} \mathcal{M}\left(\|v(\cdot, s)\|_{L^{2}}\right) d s\right) d t \geq \frac{\bar{m}_{0}\left(1-e^{-T \lambda_{n}}\right)}{\lambda_{n}} .
$$

From two estimates above, we find that

$$
\begin{aligned}
\bar{J}_{1} & \leq \frac{2 \alpha^{2} \bar{m}_{0}^{2}}{\beta^{4}}\left(\sum_{n=1}^{\infty} \frac{\lambda_{n}^{2 r+4}}{\left(1-e^{\left.-T \lambda_{n}\right)^{2}}\right.} \psi_{n}^{2}\right)\left(\int_{0}^{t}\|u-v\|_{L^{2}}^{2} d s\right) \\
& \leq \frac{2 \alpha^{2} \bar{m}_{0}^{2}}{\beta^{4}\left(1-e^{\left.-T \lambda_{1}\right)^{2}}\right.}\|\psi\|_{D\left(\mathcal{A}^{r+2}\right)}^{2}\|u-v\|_{r}^{2} \\
& \leq T C_{2}^{2}\|\psi\|_{D\left(\mathcal{A}^{r+2}\right)}^{2}\|u-v\|_{r}^{2} .
\end{aligned}
$$

By a similar argument as above, we can obtain that

$$
\bar{J}_{2} \leq T C_{3}^{2}\|\psi\|_{D\left(\mathcal{A}^{r+2}\right)}^{2}\|u-v\|_{r}^{2}
$$

Combining (3.12), (3.15), and (3.16) yields that

$$
\left\|\Phi_{1}(t) u-\Phi_{1}(t) v\right\|_{D\left(\mathcal{A}^{r}\right)}^{2} \leq T\left(C_{2}^{2}+C_{3}^{2}\right)\|\psi\|_{D\left(\mathcal{A}^{r+2}\right)}^{2}\|u-v\|_{r}^{2} .
$$

Since the left-hand side of (3.17) is independent of $t$, we arrive at

$$
\left\|\Phi_{1} u-\Phi_{1} v\right\|_{r}^{2} \leq T\left(C_{2}^{2}+C_{3}^{2}\right)\|\psi\|_{D\left(\mathcal{A}^{r+2}\right)}^{2}\|u-v\|_{r}^{2} .
$$

By letting $T$ be small enough, we get that $\Phi_{1}$ is a contraction mapping on $L^{\infty}\left(0, T ; D\left(\mathcal{A}^{r}\right)\right)$. By a similar argument as in Part 1, we can conclude that problem (3.1) has a unique solution $u \in L^{\infty}\left(0, T ; D\left(\mathcal{A}^{r}\right)\right)$. 


\section{The existence of a mild solution in the inhomogeneous case}

In this section, we focus on the existence of the mild solution of the inhomogeneous problem in the simple case $\alpha=0, \beta>0$. The proof of the second case $\alpha>0, \beta>0$ is more delicate, and we can treat it in a similar way. Hence, we do not consider it here.

Theorem 4.1 Let $\alpha=0, \beta>0$ and $\psi \in D\left(\mathcal{A}^{r+2}\right)$. If $T$ is small enough, problem (3.1) has a unique solution $u \in L^{\infty}\left(0, T ; D\left(\mathcal{A}^{r}\right)\right)$.

Proof Now, we estimate the term $\left\|\Phi_{2} u-\Phi_{2} v\right\|_{L^{\infty}\left(0, T ; D\left(\mathcal{A}^{r}\right)\right)}$ in the case $\alpha=0, \beta>0$. Let us set

$$
\begin{aligned}
& \bar{H}_{1, n}(u)=\exp \left(-\lambda_{n} \int_{0}^{t} \mathcal{M}\left(\|u(\cdot, s)\|_{L^{2}}\right) d s\right) \\
& \bar{H}_{2, n}(u)=\int_{0}^{T} \int_{0}^{t} \exp \left(-\lambda_{n} \int_{s}^{t} \mathcal{M}\left(\|u(\cdot, \eta)\|_{L^{2}}\right) d \eta\right) F_{n}(s) d s d t, \\
& \bar{H}_{3, n}(u)=\int_{0}^{T} \exp \left(-\lambda_{n} \int_{0}^{t} \mathcal{M}\left(\|u(\cdot, s)\|_{L^{2}}\right) d s\right) d t .
\end{aligned}
$$

It is easy to see that

$$
\begin{aligned}
& \left\|\Phi_{2} u-\Phi_{2} v\right\|_{D\left(\mathcal{A}^{r}\right)}^{2} \\
& =\sum_{n=1}^{\infty} \lambda_{n}^{2 r}\left(\frac{\bar{H}_{1, n}(u) \bar{H}_{2, n}(u)}{\bar{H}_{3, n}(u)}-\frac{\bar{H}_{1, n}(v) \bar{H}_{2, n}(v)}{\bar{H}_{3, n}(v)}\right)^{2} \\
& =\sum_{n=1}^{\infty} \lambda_{n}^{2 r}\left(\frac{\bar{H}_{1, n}(u) \bar{H}_{2, n}(u) \bar{H}_{3, n}(v)-\bar{H}_{1, n}(v) \bar{H}_{2, n}(v) \bar{H}_{3, n}(u)}{\bar{H}_{3, n}(u) \bar{H}_{3, n}(v)}\right)^{2} .
\end{aligned}
$$

From (3.13) and (3.14), we deduce that

$$
\bar{H}_{3, n}(u) \bar{H}_{3, n}(v) \geq\left(\frac{\bar{m}_{0}\left(1-e^{-T \lambda_{n}}\right)}{\lambda_{n}}\right)^{2}
$$

which allows us to obtain that

$$
\begin{aligned}
& \left\|\Phi_{2} u-\Phi_{2} v\right\|_{D\left(\mathcal{A}^{r}\right)}^{2} \\
& \quad \leq \frac{1}{\bar{m}_{0}^{2}\left(1-e^{-T \lambda_{1}}\right)^{2}} \sum_{n=1}^{\infty} \lambda_{n}^{2 r+2}\left(\bar{H}_{1, n}(u) \bar{H}_{2, n}(u) \bar{H}_{3, n}(v)-\bar{H}_{1, n}(v) \bar{H}_{2, n}(v) \bar{H}_{3, n}(u)\right)^{2} \\
& \quad \leq \frac{2}{\bar{m}_{0}^{2}\left(1-e^{-T \lambda_{1}}\right)^{2}} \sum_{n=1}^{\infty} \lambda_{n}^{2 r+2}\left|\bar{J}_{1, n}\right|^{2}+\frac{2}{\bar{m}_{0}^{2}\left(1-e^{-T \lambda_{1}}\right)^{2}} \sum_{n=1}^{\infty} \lambda_{n}^{2 r+2}\left|\bar{J}_{2, n}\right|^{2},
\end{aligned}
$$

where we note that

$$
\begin{aligned}
\left|\bar{H}_{1, n}(u) \bar{H}_{2, n}(u) \bar{H}_{3, n}(v)-\bar{H}_{1, n}(v) \bar{H}_{2, n}(v) \bar{H}_{3, n}(u)\right| & \\
\leq & \underbrace{\left|\bar{H}_{1, n}(u) \bar{H}_{2, n}(u)\right|\left|\bar{H}_{3, n}(v)-\bar{H}_{3, n}(u)\right|}_{\bar{J}_{1, n}} \\
& +\underbrace{\left|\bar{H}_{3, n}(u)\right|\left|\bar{H}_{1, n}(u) \bar{H}_{2, n}(u)-\bar{H}_{1, n}(v) \bar{H}_{2, n}(v)\right|}_{\bar{J}_{2, n}} .
\end{aligned}
$$


First, we have the following estimate:

$$
\begin{aligned}
&\left|\bar{H}_{3, n}(v)-\bar{H}_{3, n}(u)\right| \mid \int_{0}^{T} \exp \left(-\lambda_{n} \int_{0}^{t} \mathcal{M}\left(\|u(\cdot, s)\|_{L^{2}}\right) d s\right) d t \\
& \quad-\int_{0}^{T} \exp \left(-\lambda_{n} \int_{0}^{t} \mathcal{M}\left(\|v(\cdot, s)\|_{L^{2}}\right) d s\right) d t \mid \\
& \leq K \lambda_{n}\left(\int_{0}^{T} \int_{0}^{t}\|u(\cdot, s)-v(\cdot, s)\|_{L^{2}} d s d t\right) .
\end{aligned}
$$

Hence, we easily see that

$$
\begin{aligned}
\lambda_{n}^{2 r+2}\left|\bar{J}_{1, n}\right|^{2} \leq & \lambda_{n}^{2 r+4}\left|\bar{H}_{2, n}(u)\right|^{2} K^{2}\left(\int_{0}^{T} \int_{0}^{t}\|u(\cdot, s)-v(\cdot, s)\|_{L^{2}} d s d t\right)^{2} \\
\leq & \frac{K^{2} T^{2}}{2}\|u-v\|_{r}^{2} \lambda_{n}^{2 r+4}\left|\bar{H}_{2, n}(u)\right|^{2} \\
\leq & \frac{K^{2} T^{2}}{2}\|u-v\|_{r}^{2} \lambda_{n}^{2 r+4} \\
& \times\left|\int_{0}^{T} \int_{0}^{t} \exp \left(-\lambda_{n} \int_{s}^{t} \mathcal{M}\left(\|u(\cdot, \eta)\|_{L^{2}}\right) d \eta\right) F_{n}(s) d s d t\right|^{2},
\end{aligned}
$$

where we observe that

$$
\left|\bar{H}_{1, n}(u)\right| \leq 1 \text {. }
$$

It is not difficult to check that

$$
\begin{aligned}
& \left|\int_{0}^{T} \int_{0}^{t} \exp \left(-\lambda_{n} \int_{s}^{t} \mathcal{M}\left(\|u(\cdot, \eta)\|_{L^{2}}\right) d \eta\right) F_{n}(s) d s d t\right|^{2} \\
& \quad \leq T \int_{0}^{T}\left(\int_{0}^{t} \exp \left(-2 \lambda_{n} \int_{s}^{t} \mathcal{M}\left(\|u(\cdot, \eta)\|_{L^{2}}\right) d \eta\right) d s\right)\left(\int_{0}^{t} F_{n}^{2}(s) d s\right) d t \\
& \quad \leq T^{2} \int_{0}^{T}\left(\int_{0}^{t} F_{n}^{2}(s) d s\right) d t .
\end{aligned}
$$

Combining (4.7) and (4.8), we get

$$
\begin{aligned}
\sum_{n=1}^{\infty} \lambda_{n}^{2 r+2}\left|\bar{J}_{1, n}\right|^{2} & \leq \frac{K^{2} T^{4}}{2}\|u-v\|_{r}^{2} \int_{0}^{T}\left(\int_{0}^{t} \sum_{n=1}^{\infty} \lambda_{n}^{2 r+2} F_{n}^{2}(s) d s\right) d t \\
& \leq \frac{K^{2} T^{5}}{2}\|u-v\|_{r}^{2}\|F\|_{L^{2}\left(0, T ; D\left(\mathcal{A}^{r+1}\right)\right)}^{2} .
\end{aligned}
$$

Now it is obvious that

$$
\begin{aligned}
\left|\bar{H}_{1, n}(u) \bar{H}_{2, n}(u)-\bar{H}_{1, n}(v) \bar{H}_{2, n}(v)\right| \leq & \left|\bar{H}_{1, n}(u)\right|\left|\bar{H}_{2, n}(u)-\bar{H}_{2, n}(v)\right| \\
& +\left|\bar{H}_{2, n}(v)\right|\left|\bar{H}_{1, n}(u)-\bar{H}_{1, n}(v)\right| .
\end{aligned}
$$


So, since $\bar{H}_{3, n}(u) \leq T$ and $\bar{H}_{1, n}(u) \leq 1$, by using $(a+b)^{2} \leq 2 a^{2}+2 b^{2}$, we derive

$$
\begin{aligned}
\lambda_{n}^{2 r+2}\left|\bar{J}_{2, n}\right|^{2} \leq & T^{2} \lambda_{n}^{2 r+2}\left|\bar{H}_{1, n}(u) \bar{H}_{2, n}(u)-\bar{H}_{1, n}(v) \bar{H}_{2, n}(v)\right|^{2} \\
\leq & 2 T^{2} \lambda_{n}^{2 r+2}\left|\bar{H}_{2, n}(u)-\bar{H}_{2, n}(v)\right|^{2} \\
& +2 T^{2} \lambda_{n}^{2 r+2}\left|\bar{H}_{2, n}(v)\right|^{2}\left|\bar{H}_{1, n}(u)-\bar{H}_{1, n}(v)\right|^{2} .
\end{aligned}
$$

Let us continue and give the following bound:

$$
\begin{aligned}
\left|\bar{H}_{2, n}(u)-\bar{H}_{2, n}(v)\right|^{2} & \mid \int_{0}^{T} \int_{0}^{t} \exp \left(-\lambda_{n} \int_{s}^{t} \mathcal{M}\left(\|u(., \eta)\|_{L^{2}}\right) d \eta\right) F_{n}(s) d s d t \\
& \quad-\left.\int_{0}^{T} \int_{0}^{t} \exp \left(-\lambda_{n} \int_{s}^{t} \mathcal{M}\left(\|v(., \eta)\|_{L^{2}}\right) d \eta\right) F_{n}(s) d s d t\right|^{2} \\
\leq & T \lambda_{n}^{2} \int_{0}^{T}\left(\int_{0}^{t}\|u(\cdot, s)-v(\cdot, s)\|_{L^{2}}^{2} d s\right)\left(\int_{0}^{t} F_{n}^{2}(s) d s\right) d t \\
\leq & T^{2}\|u-v\|_{r}^{2} \int_{0}^{T} \int_{0}^{t} \lambda_{n}^{2} F_{n}^{2}(s) d s d t .
\end{aligned}
$$

This implies that

$$
\begin{aligned}
\sum_{n=1}^{\infty} \lambda_{n}^{2 r+2}\left|\bar{H}_{2, n}(u)-\bar{H}_{2, n}(v)\right|^{2} & \leq T^{2}\|u-v\|_{r}^{2} \int_{0}^{T} \int_{0}^{t}\|F(\cdot, s)\|_{D\left(\mathcal{A}^{r+1}\right)}^{2} d s d t \\
& \leq T^{3}\|u-v\|_{r}^{2}\|F\|_{L^{2}\left(0, T ; D\left(\mathcal{A}^{r+1}\right)\right)}^{2}
\end{aligned}
$$

From (3.5), we recall the following estimate:

$$
\left|\bar{H}_{1, n}(u)-\bar{H}_{1, n}(v)\right|^{2} \leq T K^{2} \lambda_{n}^{2}\|u-v\|_{r}^{2}
$$

which allows us to get that

$$
\begin{aligned}
\sum_{n=1}^{\infty} & \lambda_{n}^{2 r+2}\left|\bar{H}_{2, n}(v)\right|^{2}\left|\bar{H}_{1, n}(u)-\bar{H}_{1, n}(v)\right|^{2} \\
\leq & T K^{2} \lambda_{n}^{2 r+4}\|u-v\|_{r}^{2}\left|\int_{0}^{T} \int_{0}^{t} \exp \left(-\lambda_{n} \int_{s}^{t} \mathcal{M}\left(\|u(\cdot, \eta)\|_{L^{2}}\right) d \eta\right) F_{n}(s) d s d t\right|^{2} \\
\leq & T^{2} K^{2} \lambda_{n}^{2 r+4}\|u-v\|_{r}^{2} \\
& \times \int_{0}^{T}\left(\int_{0}^{t} \exp \left(-2 \lambda_{n} \int_{s}^{t} \mathcal{M}\left(\|u(\cdot, \eta)\|_{L^{2}}\right) d \eta\right) d s\right)\left(\int_{0}^{t} F_{n}^{2}(s) d s\right) d t \\
\leq & T^{3} K^{2}\|u-v\|_{r}^{2} \int_{0}^{T}\left(\sum_{n=1}^{\infty} \lambda_{n}^{2 r+4} \int_{0}^{t} F_{n}^{2}(s) d s\right) d t \\
\leq & T^{4} K^{2}\|u-v\|_{r}^{2}\|F\|_{L^{2}\left(0, T ; D\left(\mathcal{A}^{r+2}\right)\right)}^{2}
\end{aligned}
$$


Combining (4.11), (4.13), and (4.15), we find that

$$
\begin{aligned}
\sum_{n=1}^{\infty} \lambda_{n}^{2 r+2}\left|\bar{J}_{2, n}\right|^{2} \leq & 2 T^{2} \sum_{n=1}^{\infty} \lambda_{n}^{2 r+2}\left|\bar{H}_{2, n}(u)-\bar{H}_{2, n}(v)\right|^{2}+ \\
& +T^{2} \sum_{n=1}^{\infty} \lambda_{n}^{2 r+2}\left|\bar{H}_{2, n}(v)\right|^{2}\left|\bar{H}_{1, n}(u)-\bar{H}_{1, n}(v)\right|^{2} \\
\leq & 2 T^{5}\|u-v\|_{r}^{2}\|F\|_{L^{2}\left(0, T ; D\left(\mathcal{A}^{r+1}\right)\right)}^{2} \\
& +T^{6} K^{2}\|u-v\|_{r}^{2}\|F\|_{L^{2}\left(0, T ; D\left(\mathcal{A}^{r+2}\right)\right)}^{2} .
\end{aligned}
$$

It follows from (4.4) that

$$
\begin{aligned}
\left\|\Phi_{2} u-\Phi_{2} v\right\|_{D\left(\mathcal{A}^{r}\right)}^{2} \leq & \frac{2}{\bar{m}_{0}^{2}\left(1-e^{-T \lambda_{1}}\right)^{2}} \sum_{n=1}^{\infty} \lambda_{n}^{2 r+2}\left|\bar{J}_{1, n}\right|^{2}+\frac{2}{\bar{m}_{0}^{2}\left(1-e^{-T \lambda_{1}}\right)^{2}} \sum_{n=1}^{\infty} \lambda_{n}^{2 r+2}\left|\bar{J}_{2, n}\right|^{2} \\
\leq & \frac{4 T^{5}}{\bar{m}_{0}^{2}\left(1-e^{-T \lambda_{1}}\right)^{2}}\|u-v\|_{r}^{2}\|F\|_{L^{2}\left(0, T ; D\left(\mathcal{A}^{r+1}\right)\right)}^{2} \\
& +\frac{4 T^{6} K^{2}}{\bar{m}_{0}^{2}\left(1-e^{-T \lambda_{1}}\right)^{2}}\|u-v\|_{r}^{2}\|F\|_{L^{2}\left(0, T ; D\left(\mathcal{A}^{r+2}\right)\right)^{2}}^{2}
\end{aligned}
$$

The inequality $\sqrt{a^{2}+b^{2}} \leq a+b$ for any $a, b \geq 0$ implies that

$$
\begin{aligned}
\left\|\Phi_{2} u-\Phi_{2} v\right\|_{D\left(\mathcal{A}^{r}\right) \leq} & \frac{2 T^{5 / 2}}{\bar{m}_{0}\left(1-e^{-T \lambda_{1}}\right)}\|u-v\|_{r}^{2}\|F\|_{L^{2}\left(0, T ; D\left(\mathcal{A}^{r+1}\right)\right)}^{2} \\
& +\frac{2 T^{3} K}{\bar{m}_{0}\left(1-e^{-T \lambda_{1}}\right)}\|u-v\|_{r}\|F\|_{L^{2}\left(0, T ; D\left(\mathcal{A}^{r+2}\right)\right)} .
\end{aligned}
$$

Next, we continue to estimate the term $\left\|\Phi_{3} u-\Phi_{3} v\right\|_{D\left(\mathcal{A}^{r}\right)}$. It is obvious that

$$
\begin{aligned}
& \mid \int_{0}^{t} \exp \left(-\lambda_{n} \int_{s}^{t} \mathcal{M}\left(\|u(\cdot, \eta)\|_{L^{2}}\right) d \eta\right) F_{n}(s) d s d t \\
& \quad-\left.\int_{0}^{t} \exp \left(-\lambda_{n} \int_{s}^{t} \mathcal{M}\left(\|v(\cdot, \eta)\|_{L^{2}}\right) d \eta\right) F_{n}(s) d s d t\right|^{2} \\
& \quad \leq \lambda_{n}^{2}\left(\int_{0}^{t}\|u(\cdot, s)-v(\cdot, s)\|_{L^{2}}^{2} d s\right)\left(\int_{0}^{t} F_{n}^{2}(s) d s\right) d t \\
& \quad \leq T^{2}\|u-v\|_{r}^{2} \int_{0}^{t} \lambda_{n}^{2} F_{n}^{2}(s) d s .
\end{aligned}
$$

Hence, we derive the following estimate:

$$
\begin{aligned}
\left\|\Phi_{3} u-\Phi_{3} v\right\|_{D\left(\mathcal{A}^{r}\right)}^{2} & \sum_{n=1}^{\infty} \lambda_{n}^{2 r} \mid \int_{0}^{t} \exp \left(-\lambda_{n} \int_{s}^{t} \mathcal{M}\left(\|u(\cdot, \eta)\|_{L^{2}}\right) d \eta\right) F_{n}(s) d s d t \\
& -\left.\int_{0}^{t} \exp \left(-\lambda_{n} \int_{s}^{t} \mathcal{M}\left(\|v(\cdot, \eta)\|_{L^{2}}\right) d \eta\right) F_{n}(s) d s d t\right|^{2}
\end{aligned}
$$




$$
\begin{aligned}
& \leq T^{2}\|u-v\|_{r}^{2} \int_{0}^{t} \sum_{n=1}^{\infty} \lambda_{n}^{2 r+2} F_{n}^{2}(s) d s \\
& =T^{2}\|u-v\|_{r}^{2}\|F\|_{L^{2}\left(0, T ; D\left(\mathcal{A}^{r+1}\right)\right)}^{2},
\end{aligned}
$$

which allows us to obtain that

$$
\left\|\Phi_{3} u-\Phi_{3} v\right\|_{D\left(\mathcal{A}^{r}\right)} \leq T\|u-v\|_{r}\|F\|_{L^{2}\left(0, T ; D\left(\mathcal{A}^{r+1}\right)\right)} .
$$

Combining (3.18), (4.18), and (4.21), we find that

$$
\begin{aligned}
\|\Phi u-\Phi v\|_{r} \leq & \left\|\Phi_{1} u-\Phi_{1} v\right\|_{D\left(\mathcal{A}^{r}\right)}+\left\|\Phi_{2} u-\Phi_{2} v\right\|_{D\left(\mathcal{A}^{r}\right)}+\left\|\Phi_{3} u-\Phi_{3} v\right\|_{D\left(\mathcal{A}^{r}\right)} \\
\leq & \sqrt{T\left(C_{2}^{2}+C_{3}^{2}\right)}\|\psi\|_{D\left(\mathcal{A}^{r+2}\right)}\|u-v\|_{r}+T\|u-v\|_{r}\|F\|_{L^{2}\left(0, T ; D\left(\mathcal{A}^{r+1}\right)\right)} \\
& +\frac{2 T^{5 / 2}}{\bar{m}_{0}\left(1-e^{-T \lambda_{1}}\right)}\|u-v\|_{r}^{2}\|F\|_{L^{2}\left(0, T ; D\left(\mathcal{A}^{r+1}\right)\right)}^{2} \\
& +\frac{2 T^{3} K}{\bar{m}_{0}\left(1-e^{\left.-T \lambda_{1}\right)}\right.}\|u-v\|_{r}\|F\|_{L^{2}\left(0, T ; D\left(\mathcal{A}^{r+2}\right)\right)} .
\end{aligned}
$$

From the latter estimate, we can find $T$ small enough such that $\Phi_{1}$ is a contraction mapping on $L^{\infty}\left(0, T ; D\left(\mathcal{A}^{r}\right)\right)$.

\section{III-posedness of the mild solution}

If $\alpha=0$ and if $\psi \in D(\mathcal{A})$, the mild solution of problem (1.1) is given by the integral equation

$$
u(t)=\sum_{n=1}^{\infty} \frac{\exp \left(-\lambda_{n} \int_{0}^{t} \mathcal{M}\left(\|u(\cdot, s)\|_{L^{2}}\right) d s\right)\left\langle\psi, \varphi_{n}\right\rangle}{\beta \int_{0}^{T} \exp \left(-\lambda_{n} \int_{0}^{t} \mathcal{M}\left(\|u(\cdot, s)\|_{L^{2}}\right) d s\right) d t} \varphi_{n} .
$$

Assume that $\psi^{0}=0$ then $u^{*}=0$. For $m \in \mathbb{N}$, let $\bar{\psi}_{m}$ be measured data which satisfy

$$
\bar{\psi}_{m}(x)=\psi^{0}(x)+\frac{1}{\sqrt{\lambda_{m}}} \varphi_{m}(x)=\frac{1}{\sqrt{\lambda_{m}}} \varphi_{m}(x)
$$

It is easy to see that $\bar{\psi}_{m} \in D(\mathcal{A})$ and the following fact holds:

$$
\left\|\bar{\psi}^{m}-\psi^{0}\right\|_{L^{2}}=\frac{1}{\sqrt{\lambda_{m}}} \rightarrow 0, \quad m \rightarrow \infty .
$$

The mild solution of problem (1.1) corresponding to $\bar{\psi}_{m}$ is

$$
\begin{aligned}
u_{m}(t) & =\sum_{n=1}^{\infty} \frac{\exp \left(-\lambda_{n} \int_{0}^{t} \mathcal{M}\left(\left\|u_{m}(\cdot, s)\right\|_{L^{2}}\right) d s\right)\left\langle\bar{\psi}^{m}, \varphi_{n}\right\rangle}{\beta \int_{0}^{T} \exp \left(-\lambda_{n} \int_{0}^{t} \mathcal{M}\left(\left\|u_{m}(\cdot, s)\right\|_{L^{2}}\right) d s\right) d t} \varphi_{n} \\
& =\frac{\exp \left(-\lambda_{m} \int_{0}^{t} \mathcal{M}\left(\left\|u_{m}(\cdot, s)\right\|_{L^{2}}\right) d s\right)}{\beta \sqrt{\lambda_{m}} \int_{0}^{T} \exp \left(-\lambda_{m} \int_{0}^{t} \mathcal{M}\left(\left\|u_{m}(\cdot, s)\right\|_{L^{2}}\right) d s\right) d t} \varphi_{m} .
\end{aligned}
$$

It implies immediately that

$$
\left\|u_{m}(\cdot, 0)\right\|_{L^{2}}=\frac{1}{\beta \sqrt{\lambda_{m}} \int_{0}^{T} \exp \left(-\lambda_{m} \int_{0}^{t} \mathcal{M}\left(\left\|u_{m}(\cdot, s)\right\|_{L^{2}}\right) d s\right) d t} .
$$


Let us emphasize that

$$
\begin{aligned}
\int_{0}^{T} \exp \left(-\lambda_{m} \int_{0}^{t} \mathcal{M}\left(\left\|u_{m}(\cdot, s)\right\|_{L^{2}}\right) d s\right) d t & \geq \int_{0}^{T} \exp \left(-\lambda_{m} \int_{0}^{t} \bar{m}_{0} d s\right) d t \\
& =\frac{1-e^{-\bar{m}_{0} \lambda_{m} T}}{m_{0} \lambda_{m}} .
\end{aligned}
$$

Hence, we get the following estimate:

$$
\begin{aligned}
\left\|u_{m}(\cdot, 0)\right\|_{L^{2}} & =\left\|u_{m}(\cdot, 0)-u^{*}(0)\right\|_{L^{2}} \\
& \geq \frac{m_{0} \lambda_{m}}{1-e^{-\bar{m}_{0} \lambda_{m} T}} \frac{1}{\beta \sqrt{\lambda_{m}}} \\
& =\frac{m_{0} \sqrt{\lambda_{m}}}{\beta\left(1-e^{-\bar{m}_{0} \lambda_{m} T}\right)} .
\end{aligned}
$$

From the fact that

$$
\lim _{m \rightarrow \infty} \frac{m_{0} \sqrt{\lambda_{m}}}{\beta\left(1-e^{-\bar{m}_{0} \lambda_{m} T}\right)}=\infty
$$

we deduce

$$
\lim _{m \rightarrow \infty}\left\|u_{m}(\cdot, 0)-u^{*}(0)\right\|_{L^{2}}=\infty
$$

\section{Acknowledgements}

Not applicable.

\section{Funding}

This research is funded by Thu Dau Mot University under grant number DT.20.2-039.

\section{Availability of data and materials}

Not applicable.

\section{Competing interests}

The authors declare no conflict of interest.

\section{Authors' contributions}

All authors contributed equally. All the authors read and approved the final manuscript.

\section{Author details}

'Division of Applied Mathematics, Thu Dau Mot University, Binh Duong Province, Vietnam. ${ }^{2}$ Department of Mathematics, Faculty of Arts and Sciences, Cankaya University, 06530 Ankara, Turkey. ${ }^{3}$ Department of Medical Research, China Medical University Hospital, China Medical University, Taichung, Taiwan. ${ }^{4}$ Institute of Space Sciences, P.O. Box, MG-23, R 76900, Magurele-Bucharest, Romania. ${ }^{5}$ Institute of Research and Development, Duy Tan University, Da Nang, Vietnam. ${ }^{6}$ Applied Analysis Research Group, Faculty of Mathematics and Statistics, Ton Duc Thang University, Ho Chi Minh City, Vietnam.

\section{Publisher's Note}

Springer Nature remains neutral with regard to jurisdictional claims in published maps and institutional affiliations.

Received: 27 July 2020 Accepted: 21 October 2020 Published online: 31 October 2020

References

1. Gobbino, M.: Quasilinear degenerate parabolic equations of Kirchhoff type. Math. Methods Appl. Sci. 22(5), 375-388 (1999)

2. Gobbino, M.: Quasilinear degenerate parabolic equation of Kirchhoff type. Math. Methods Appl. Sci. 22, 375-388 (1999)

3. Tudorascu, A., Wunsch, M.: On a nonlinear, nonlocal parabolic problem with conservation of mass, mean and variance. Commun. Partial Differ. Equ. 36, 1426-1454 (2011) 
4. Ghist, M., Gobbino, M.: Hyperbolic-parabolic singular perturbation for mildly degenerate Kirchhoff equations: time-decay estimates. J. Differ. Equ. 245, 2979-3007 (2008)

5. Hashimoto, M., Yamazaki, T.: Hyperbolic-parabolic singular perturbation for quasilinear equations of Kirchhoff type. J. Differ. Equ. 237, 491-525 (2007)

6. Autuori, G., Pucci, P., Salvatori, M.C.: Asymptotic stability for anisotropic Kirchhoff systems. J. Math. Anal. Appl. 352, 149-165 (2009)

7. Chang, N.H., Chipot, M.: Nonlinear nonlocal evolution problems. Rev. R. Acad. Cienc. Exactas Fís. Nat., Ser. A Mat. 97, 393-415 (2003)

8. Chipot, M., Lovat, B.: Some remarks on nonlocal elliptic and parabolic problems. In: Proceedings of the Second World Congress of Nonlinear Analysts, Part 7, Athens, 1996, vol. 30, pp. 4619-4627 (1997)

9. Jorge, J.F., de Oliveira, H.B.: Parabolic reaction-diffusion systems with nonlocal coupled diffusivity terms. Discrete Contin. Dyn. Syst. 37(5), 2431-2453 (2017)

10. Tuan, N.H., Au, V.V., Khoa, V.A., Lesnic, D.: Identification of the population density of a species model with nonlocal diffusion and nonlinear reaction. Inverse Probl. 33, 5 (2017)

11. Tuan, N.H., Nam, D.H.Q., Nhat, V.T.M.: On a backward problem for the Kirchhoff's model of parabolic type. Comput. Math. Appl. 77(1), 15-33 (2019)

12. Dokuchaev, N.: On recovering parabolic diffusions from their time-averages. Calc. Var. Partial Differ. Equ. 58(1), 27 (2019)

13. Dokuchaev, N.: Regularity of complexified hyperbolic wave equations with integral conditions. https://arxiv.org/abs/1907.03527

14. Volodymyr, S.I., Nytrebych, Z.M., Pukach, P.Y.: Boundary-value problems with integral conditions for a system of Lamé equations in the space of almost periodic functions. Electron. J. Differ. Equ. 2016(304), 1 (2016)

15. Pulkina, S.L., Savenkova, A.E.: A problem with a nonlocal, with respect to time, condition for multidimensional hyperbolic equations. Russ. Math. 60(10), 33-43 (2016)

16. Agarwal, P., Baltaeva, U., Alikulov, Y.: Solvability of the boundary-value problem for a linear loaded integro-differential equation in an infinite three-dimensional domain. Chaos Solitons Fractals 140, 110108 (2020)

17. Agarwal, P., Akbar, M., Nawaz, R., Jleli, M.: Solutions of system of Volterra integro-differential equations using optimal homotopy asymptotic method. Math. Methods Appl. Sci. https://doi.org/10.1002/mma.6783

18. Kaur, D., Agarwal, P., Rakshit, M., Chand, M.: Fractional calculus involving $(p, q)$-Mathieu type series. Appl. Math Nonlinear Sci. 5(2), 15-34 (2020)

19. Agarwal, P., Attary, M., Maghasedi, M., Kumam, P.: Solving higher-order boundary and initial value problems via Chebyshev spectral method: application in elastic foundation. Symmetry 12, 987 (2020)

20. Caraballo, T., Cobos, M.H., Rubio, P.M.: Long-time behavior of a non-autonomous parabolic equation with nonlocal diffusion and sublinear terms. Nonlinear Anal. 121, 3-18 (2015)

21. Cao, C., Rammaha, M.A., Titi, E.S.: The Navier-Stokes equations on the rotating 2-D sphere: Gevrey regularity and asymptotic degrees of freedom. Z. Angew. Math. Phys. 50, 341-360 (1999)

22. Dawidowski, L.: The quasilinear parabolic Kirchhoff equation. Open Math. 15, 382-392 (2017)

23. Ferreira, J., Oliveira, B.H.: Parabolic reaction-diffusion systems with nonlocal coupled diffusivity terms. Discrete Contin. Dyn. Syst. 37(5), 2431-2453 (2017)

24. Figueiredo, M.G., Ikoma, S.N., Santos, R.J.J.: Existence and concentration result for the Kirchhoff type equations with general nonlinearities. Arch. Ration. Mech. Anal. 213(3), 931-979 (2014)

25. Fu, Y., Xiang, M.: Existence of solutions for parabolic equations of Kirchhoff type involving variable exponent. Appl. Anal. 95(3), 524-544 (2016)

26. Tuan, N.H., Baleanu, D., Thach, T.N., O'Regan, D., Can, N.H.: Final value problem for nonlinear time fractional reaction-diffusion equation with discrete data. J. Comput. Appl. Math. 376, 112883 (2020)

27. Caraballo, T., Herrera-Cobos, M.C., Marin, P.R.: Global attractor for a nonlocal $p$-Laplacian equation without uniqueness of solution. Discrete Contin. Dyn. Syst., Ser. B 22(5), 1801-1816 (2017)

\section{Submit your manuscript to a SpringerOpen ${ }^{\circ}$ journal and benefit from:}

- Convenient online submission

- Rigorous peer review

- Open access: articles freely available online

- High visibility within the field

- Retaining the copyright to your article

Submit your next manuscript at $\boldsymbol{~ s p r i n g e r o p e n . c o m ~}$ 